\title{
RELATING TOLERANCES AND KINEMATIC BEHAVIOR
}

\author{
D.A. Bourne, D. Navinchandra and R. Ramaswamy ${ }^{1}$
}

CMU-RI-TR-89-10

\author{
The Robotics Institute \\ Carnegie Mellon University \\ Pittsburgh, Pennsylvania 15213
}

March 1989

Copyright (C) 1989 Carnegie Mellon University

\footnotetext{
${ }^{1}$ Supported during this work by the Machinist Expert Consortium at The Robotics Institute, Camegie Mellon University
} 
Table of Contents

1. Introduction

2. Relevant Work

3. Configuration Spaces

3.1. Introduction

3.2. Basic Theory

3.3. Important Properties

4. Relating Tolerances and Kinematic Behavior

4.1. Example 1: Circuit Breaker

4.2. Example 2: Window Regulator Mechanism

5. Implementation Issues

5.1. Representation

5.2. C-space calculation

5.3. Reasoning

5.4. Current Status

6. Research Issues

7. Summary 


\section{List of Figures}

Figure 3-1: Simple Configuration Spaces 


\section{List of Tables}

Table 2-1: Sample list of behavioral features [4] 


\begin{abstract}
Designers usually complete the design with nominal dimensions and allocate tolerances only at the drawing stage. This practice can cause the following problems: (1) unnecessarily tight tolerances that require expensive manufacturing processes, (2) parts whose proper functioning is contingent on excessively tight tolerances, and (3) situations where slight wear on a part can seriously modify the bchavior of the device. There is a need for computer-based techniques which will allow designers to investigate the effect of manufacturing tolerances on the function their design performs. This paper presents a means for capluring the kinematic behavior of a device and relating it to the tolerances on it's components. Behavior is represented using a configuration space representation, which we argue will be a useful tool for designers.
\end{abstract}




\section{Introduction}

Designers usually prefer to work with exact dimensions. This is because design equations use precise values for dimensions of geometric entities (see, for example, the design text by Shigley [20]). Before a design can be manufactured, however, tolerances have to be specified on all features because no production process can make exact parts. Tolerances are also significant because their magnitude decides the manufacturing processes that can be used. This directly affects the cost of the finished part.

When nominal dimensions are used for a design, the effect of inaccuracies in the dimensions of various parts is not considered. This practice may lead to problems such as,

- The tolerances specified are smaller than existing process capabilities and have to be modified on the shop-floor before production of the part. As a result, the device produced may not perform as the designer intended.

- Parts do not fulfill their intended function even when manufactured within the specified tolerance. Unforeseen effects such as interference of parts and excessive play can occur.

- Device behavior is very sensitive to component tolerances.

Since these problems usually involve more than one part, they are often discovered late in the product cycle, possibly even after the product is put into service. The redesign, recall and warranty costs incurred strongly affect the profitability of the product. These losses can be directly linked to the inappropriate tolerances assigned during the design phase.

The accepted method of preventing tolerance problems is to rely heavily on the judgement of the designer. The designer must ensure that suitable tolerances are allocated and recorded using standard drafting practice. This is done according to detailed standards that specify the correct syntax and semantics of the symbols used. Voelcker [22] has reviewed the evolution of engineering representations and summarized some reasons for the development of modern drafting practices.

Unfortunately, designers often lack tools to aid them in tolerance allocation and hence may do this by reference to previous similar designs, intuition or other informal methods. There are several ways in which $\mathrm{CAD}$ systems could aid designers in detecting and reducing the sensitivity of designs to tolerances. Some of these are,

1. Older guides to tolerancing, like the text by Peck [15], advise that tight 
tolerances be assigned only to the "critical dimensions". However, the designer must still identify the critical dimensions. Programs that aid the designer in this task would be useful.

2. All mechanisms exhibit wear, which changes the dimensions of certain surfaces. Tools for automatically determining candidate features for high wear and its effect on device behavior can aid designers.

3. Errors such as multiply dimensioned links and excessive stackup could be detected before the drawing is transmitted for manufacturing.

In order to provide the above capabilities, we require a suitable representation which can be used to estimate the effect of dimensional tolerance on the behavior of a device. In this paper, we examine one such representation and demonstrate the utility of our approach through several examples, which could be used to guide the design of computer-aided tolerancing methods. The methods we propose are not intended to replace existing manual techniques but to provide a computer-based method of relating manufacturing tolerances and behavior. Though limited in scope, the algorithm still offers capabilities that have been unavailable in CAD systems.

An important benefit of this representation is the ability to augment the design information currently available from engineering drawings. As drawings are limited to describing geometry, it is common practice to extensively annotate the drawing with other relevant information. This practice, though sometimes useful, can be confusing. In particular, the designer's intent cannot be unambiguously recorded in footnotes. We contend that specifying tolerances on a design is a method of ensuring that when the mechanism is manufactured, it exhibits a specified behavior. Stated alternatively, the real objective is to achieve a particular behavior. Tolerances are a crude way of ensuring that the manufactured device conforms to this intent. Hence, a description of the desired kinematic behavior may be useful information to include with a design. This would then be available for reference to supplement the information provided on the drawing.

\section{Relevant Work}

Most textbooks, for example Peck [15], and the relevant ANSI standard on tolerancing ANSI-Y14.5M [2] deal extensively with methods of clearly and unambiguously representing tolerances, but largely ignore the issue of allocation. In other words, they are drafting and not 
engineering standards. The problem of tolerance allocation has not been standardized and is still the focus of research efforts.

It is sometimes possible to express the behavior of a part as a mathematical function of all its geometric parameters. In this case, determining sensitivity to tolerance reduces to taking partial derivatives. The function describing the behavior may be differentiated with respect to each dimension and the dimensions with the highest gradients can be considered as the critical ones. For example, when designing a linkage to follow a specified trajectory, it is possible to express the position of any point on the linkage as a function of the link lengths and joint angles. The sensitivity of the trajectory to inaccuracies in the link lengths can then be calculated by taking partial derivatives. Though this approach provides a rigorous way of ensuring that the dimensions chosen minimize the effect of inaccuracies on behavior, designers cannot apply it in most practical cases. The problems involved in defining behavior as a mathematical quantity and in computing its relation to the dimensions of the part cannot usually be solved.

Some researchers have attempted to perform optimal tolerance allocation based on various criteria. A common objective is minimum cost. The procedure assumes a model that relates tolerance to cost, formulates constraints on the minimum and maximum allowable tolerances on individual and concatenated dimensions and minimizes the total cost of achieving these tolerances. The tolerances used may be worst cases or statistical mean values. Optimization approaches to tolerance allocation are surveyed by Chase [3]. This paper also presents a technique for tolerance allocation when using processes that do not produce parts following an ideal Gaussian distribution. Taguchi [21] has proposed techniques for allocating tolerances that aim at maximizing the quality of a part.

Some researchers have focused on methods of representing tolerances. Requicha [18] suggests an integrated way of representing and reasoning about tolerances. He also suggests some ways of detecting inconsistencies in toleranced drawings. Hillyard [11] attempts to do this by converting the description to a graph structure and searching the graph for cycles. A cycle in the graph indicates the presence of an over-dimensioned part, i.e. an assembly that has an overall tolerance that is not consistent with the tolerances on its component parts. Hoffmann [12] has provided a linear programming formulation for this problem.

There are some areas in mechanical engineering where the tolerance on components can be 
selected based purely on the required behavior of the device. An example is the design of mating cylindrical parts [20]. The ANSI-B4.1 [1] standard specifies the clearance required to achieve a particular fit. A sample condition is,

Close sliding fits are intended for accurate location of

parts which must assemble without perceptible play.

Along with the above information, a table specifies the tolerances to be allocated for shafts and holes of various nominal sizes. This system of selecting a standard tolerance based on the function the device performs takes the uncertainty out of tolerancing once the initial data has been collected, verified and tabulated. If available on-line, information relating functions and recommended tolerances would lead to suitable tolerances being allocated in standard cases.

This methodology could possibly be extended to include a broader range of mechanical behaviors. Ideally, the designer would be able to choose an appropriate tolerance based only on the size of the feature and the desired behavior. One approach to characterizing various types of behavior is to compile lists of qualitative descriptions of the functions of mechanical components by taking apart large assemblies and analyzing each part. Though the choice of features is subjective, an acceptable list can be compiled for a restricted domain. One such analysis, presented by Collins [4], provides a set of "elemental mechanical functions" or behavioral features, based on data collected while analyzing helicopter engine failures. A representative sample of these is reproduced in Table $2-1$.

This list contains many apparent duplications due to its inability to precisely define the semantics of each category. The problem appears to be the ambiguity of the natural language used to describe the functions. It may however be possible to develop a more precise list and to recommend tolerances for each case. This would allow a designer to tolerance a part based on its function. An obstacle to such an approach is that it is difficult to pin down the exact functionality of mechanical parts. Another important issue is whether the information carried by the representation used is sufficient. Though loss of information is inevitable when switching to a qualitative scheme, a representation should still support any required reasoning. The limitations of purely qualitative representations have been examined in the literature on qualitative kinematics and statics, e.g. Forbus [9], Nielsen [14].

For the high level descriptors shown in Table 2-1, we find that lack of information is a major 


\begin{tabular}{lll}
\hline 1. Supporting & 7. Oscillatory sliding & 13. Clutching \\
2. Attaching & 8. Removable fastening & 14. Flexible spacing \\
3. Motion constraining & 9. Oscillatory rolling & 15. Deflecting \\
4. Force constraining & 10. Permanent fastening & 16. Spacing \\
5. Friction reducing & 11. Motion damping & \\
6. Pivoting & 12. Force distributing & \\
\hline
\end{tabular}

Table 2-1: Sample list of behavioral features [4]

problem. This leads us to search for more precise ways of describing the behavior of a part that would allow qualitative reasoning yet still retain all the original information.

The configuration space (c-space) representation shows the potential for supporting both quantitative and qualitative reasoning at multiple resolutions. The c-space can be used to describe all possible kinematic behaviors of a device. The remainder of this paper describes the basic theory of c-spaces and discusses examples of using this approach to analyze the sensitivity of a device's behavior to tolerance. The limitations of this approach, potential problems with its implementation and other research issues are also covered.

\section{Configuration Spaces}

\subsection{Introduction}

The term configuration space was introduced by Lozano-Perez $[16,17]$ in the domain of motion planning for robots.More recently, this representation has been applied to the analysis and design of mechanisms by Joskovicz [13] and Faltings [5].

The c-space can describe every possible placement of the links of a mechanism. A legal placement is one where links do not physically interfere. A point in the c-space is a vector of the values of the position and orientation parameters of each link. Since all placements are enumerated in the c-space, any motion can be described as a curve in the legal region of the c-space. 


\subsection{Basic Theory}

A configuration of a single object is a vector of six parameters, three positions and three orientations. The c-space consists of all possible values of this vector. Consider a mechanism with two links. If regarded individually, the two links have a total of two times six, i.e. twelve degrees of freedom. However, as two objects cannot overlap in space, some configurations become illegal. Hence, the c-space is partitioned into subsets corresponding to legal and illegal placements of the links. The illegal or forbidden region is shown as the shaded area in all the figures in this paper. Regions corresponding to legal configurations, shown as unshaded areas in the figures, are where all motions of the mechanism must occur. (The $\mathrm{c}$ spaces shown in this paper are all hand-drawn approximations.)

Some simple mechanisms have two links and each link has exactly one degree of freedom. Figure 3-1(a) shows such a mechanism where the disk is constrained to rotate about point $O$ and the rod is constrained to translate along the direction $\mathrm{L}$ indicated. The configuration vector for this mechanism thus has two elements namely $\Theta$, the angular position of the disk and $\mathrm{X}$, the linear position of the rod. Taken separately, the ranges for $\mathrm{X}$ and $\Theta$ would have been $[0,+\infty]$ and $[0,2 \pi]$. However, since the rod cannot overlap the disk, its range of motion becomes confined to the intervals $[R,+\infty]$. The range of rotations for the disk remains unaffected. The c-space is thus divided into regions corresponding to the free and forbidden placements of the links. These are depicted as the unshaded and shaded regions respectively. All motions of the mechanism must include only points in the unshaded region.

The next example, shown in Figure 3-1(b), is slightly more complicated. The form of the mechanism is similar to that of part (a) except for the addition of a projection on the disk. This introduces a change in the behavior of the device. The modified $c$-space now has a notch in the legal region. To understand why this change occurred, let us trace the sequence of events as the cam rotates in a counter clockwise direction. As the left upper tip of the projection touches the follower face, it pushes the follower upward. The $\mathrm{x}$ value of the follower then begins to increase. This is reflected by the beginning of the notch in the c-space in Figure 3-1(b). This continues until a position is reached where the top face of the projection and the bottom face of the follower are parallel and touch. The sequence of events is then reversed and the follower descends. If the height of the projection is gradually reduced, and the c-space repeatedly redrawn, it will be seen that the depth of the notch gradually reduces until the original c-space of part (a) is reached in the limiting case where 
a)
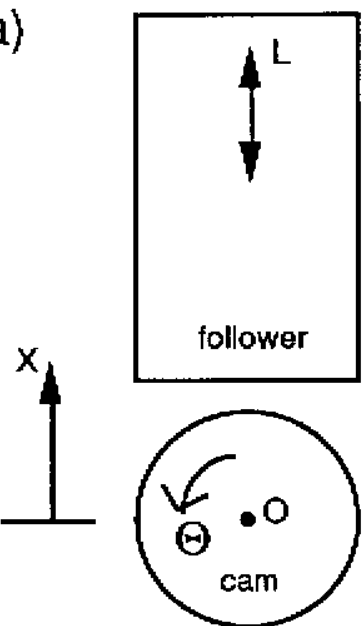

b)

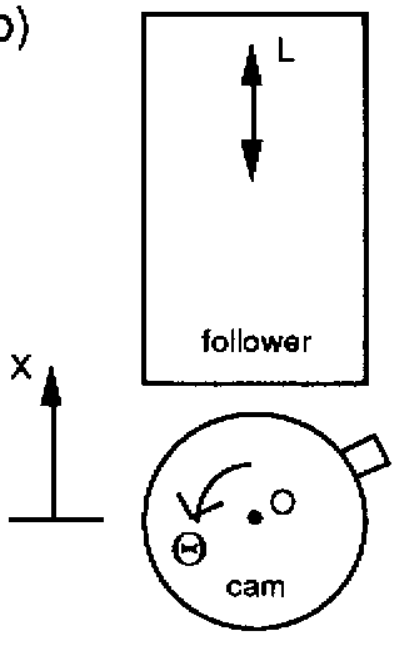

360

$\Theta$

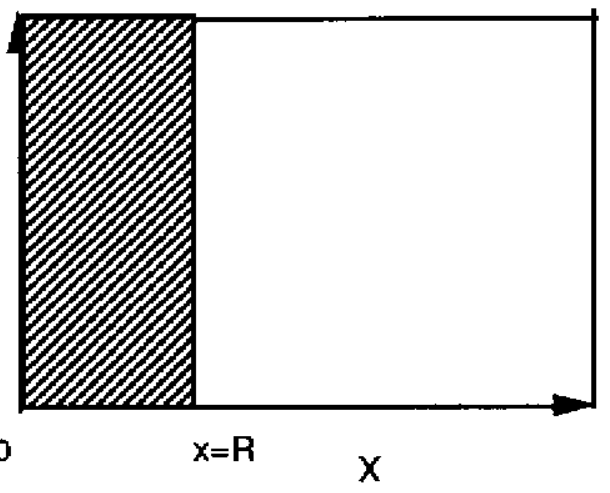

360

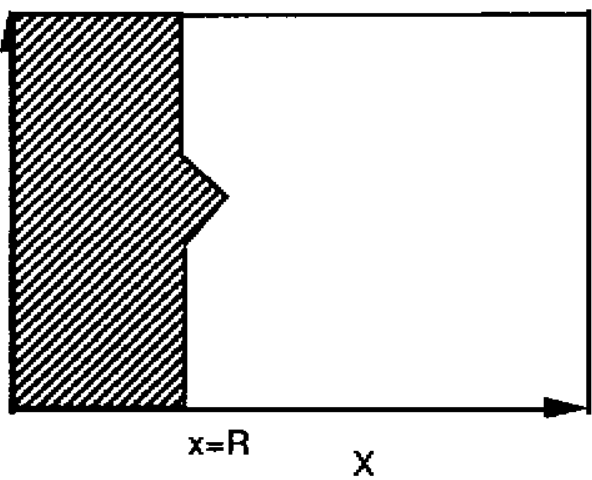

disk radius $=\mathrm{B}$

Figure 3-1: Simple Configuration Spaces

the projection has a height of zero.

It is usually impractical to calculate the entire c-space of a mechanism in a single step because of the high dimensionality of the space (6n, where $n$ is the number of links). A useful approach is to consider the mechanism to be composed of smaller functional units. The cspace for each individual unit is calculated and these are composed to give the c-space for the entire mechanism. Methods for doing this are suggested by Joskovicz [13] who rigorously 
proves the validity of this procedure. Our discussion limits itself to the domain of two-axis mechanisms because they can be analyzed without too much computation but still serve to illustrate the underlying principles.

\subsection{Important Properties}

Joskovicz [13] has identified several interesting properties of c-spaces and has provided a detailed listing of these in his thesis. The most relevant for tolerance analysis is that the $c$ space of a mechanism can be considered as the union of several connected subsets. There is a one-to-one correspondence between these subsets and possible motion types. For the fixed axis mechanisms considered by Joskovicz, the possible motions are linear, rotational and helical. Complex mechanisms consist of a closed chain of kinematic pairs (see Reuleaux [19]), which are the smallest functional sub-assemblies of the mechanism. The cspace of a kinematic chain can be calculated by composing the c-spaces of all possible kinematic pairs. A useful simplification is that only those elements that can actually come in contact need be considered. This may make it possible to handle larger mechanisms.

The qualitatively similar subsets of the c-space were referred to as regions by Joskovicz. He used the c-space as the basis for calculating a region diagram which showed all the regions and the possible transitions between them. Faltings [7] has used the similar idea of place vocabulary. This term was originally suggested by Forbus $[8,9]$ to refer to possible regions of motion of a point mass amidst polygonal obstacles. Faltings has extended the idea to cspaces. He defines a place as a set of qualitatively equivalent points in the c-space of a mechanism. Behavior can then be defined by listing all the places and the transitions between them in a representation called a place graph. He has explored methods of making a transition between the place graph and c-spaces. The concept of qualitatively similar classes of behaviors is used later in the paper to estimate the effects of tolerance.

As stated earlier, all motions of a mechanism must occur within the legal region of the cspace. Any motion is represented by a start and an end point in the c-space which are joined by a continuous curve. This curve may be arbitrarily complex but, to represent a legal motion, it must be continuous. We refer to such curves as a paths in the c-space. Most interesting paths occur along the boundary of the legal region of the c-space. Introduction of extemal tendencies such as springs cause the mechanism to follow certain paths preferentially. This is seen in the circuit-breaker example presented in the next section. 
We are trying to use $c$-spaces to analyze the quality of a design as well as its simple behavior. We are interested in how a device might wear over time and how tolerance specifications affect behavior. It is possible to predict some of the effects of wear from the c-space. As boundaries of the c-space correspond to contacts between parts of the mechanism, wear on these parts causes these boundaries to shift. Wear can therefore be interpreted as a progressive widening of the boundaries of the legal region of the c-space.

\section{Relating Tolerances and Kinematic Behavior}

One of the major aims of using a c-space approach is to provide designers with insights into the behavior of a device that are not evident from its geometry. The critical feature in this regard is that each subset of the c-space is associated with a qualitatively similar set of behaviors. A major portion of the analysis we present here relies on this basic property of topology (Euler number) of the c-space. The topology of the configuration space is a property that the designer would like to preserve since a change in topology is indicative of qualitatively different behavior. However, as seen in the following examples, if topology alone is used as a descriptor, significant changes in the behavior may be overlooked. Hence, it is necessary to also maintain some geometric information such as the number of segments constituting the boundary, the convexity of individual regions etc.

Perturbation of geometry to test for for changes in behavior has been demonstrated by Faltings [6] in his thesis but provides no method for selecting which parts of the geometry to perturb. This is significant, because exhaustively modifying each geometric parameter and all combinations thereof is not feasible. We propose the use of heuristics to analyze the c-space and determine candidate dimensions for perturbation.

Once the perturbation has been performed and the c-space recomputed, it is necessary to perform a diagnosis, i.e. to determine if a major qualitative change has occurred in the behavior. The following are some specific changes that may occur in the c-space which can used to predict unacceptable variations in the functioning of the device. They are listed in order of increasing importance.

1. Changes in the topology of the c-space. Appearance or disappearance of regions indicates that the basic type of motion is changing.

2. Changes in the boundaries of the c-space. Though topology may be unaffected, a tolerance may cause the appearance of new boundary segments. Since this 
indicates a change in the nature of contacts and hence the place vocabulary, it is probably undesirable.

3. Changes in geometric parameters. The above categories do not account for changes in the geometric properties such as the aspect ratio. This type of change may also be indicative of undesirable changes in behavior.

The c-space can be used to monitor changes in the qualitative behavior of the device, the nature of the physical contact occurring and the magnitude of relative motions along different axes. In addition, it is possible to directly perturb c-space boundaries to predict the effect of phenomenon like wear or failure. For example, to account for wear we can shift some of the c-space boundaries by a suitable amount.

The following examples illustrate some of these situations and the use of the c-space to detect problems.

\subsection{Example 1: Circuit Breaker}

This example illustrates the use of c-spaces as an aid for designers. The artifact to be designed is a circuit breaker using a bi-metallic strip. The basic principle is that the electrical circuit is maintained by contact between two links, one of which is attached to a bi-metallic strip. When the temperature rises, the bi-metallic strip deflects, thereby pulling the links apart and breaking the circuit. This state is maintained until the device is manually reset.

A typical design is shown in Figure 4-1. The electrical circuit is completed by contact between the two links L1 and L2. L1 is connected to the bi-metallic strip and can translate in the $\mathbf{X}$ direction and $\mathbf{L} 2$ rotates about $\boldsymbol{O}$. The angle of rotation is denoted as $\Theta$. A torsion spring causes $\mathbf{L} 2$ to always try and rotate clockwise.

Some of the constraints on the design are as follows,

1. The device must break the contact only when an appreciable upward movement of $\mathbf{L l}$ occurs.

2. The contact must be broken cleanly to prevent sparking, i.e. the contact must either occur over an area or not at all and point contacts are to be avoided.

We now present a scenario in which the designer starts from an initial rough design and modifies it to satisfy the behavioral specifications by reasoning about its c-space The first design attempt is shown in Figure 4-1(a). The translational axis of $\mathbf{L} \mathbf{1}$ passes through the 
center of rotation of L2. The c-space for this mechanism is shown in Figure 4-1(b).(The ranges of $\Theta X$ have been restricted to $[0,180]$ and $[0, X \mathrm{~m}]$ respectively). The initial position of the mechanism is marked a. The spring loading of L2 ensures that the state moves to the maximum value of $\Theta$ that can be reached while traveling on a line parallel to the $\Theta$ axis without passing through any illegal regions. If no such line exists, movement occurs along boundaries of the legal region that allow an increase in $\Theta$. For this design, the initial position is at the bottom of a notch. Hence as $\mathbf{X}$ increases, motion initially occurs along the segments $\mathbf{a b}$ and then, under the action of the spring, along $b c$.

The path followed by the mechanism while breaking the contact is abc. This suffers from the drawbacks that it requires only a marginal upward movement of L1 for the circuit to be broken. Hence, this mechanism is extremely sensitive to the relative vertical locations of $\mathbf{L} 1$ and L2. A small change in the vertical position of $\mathbf{L} 1$ causes the contact to be broken. When wear occurs, the sharp point $b$ in the $c$-space will become rounded and make it even easier for the mechanism to slip. In addition, the segment ab corresponds to a vertex-edge contact constraint and hence will induce sparking. As only the small horizontal faces of L1 and L2 are utilized, the contact area is quite low.

This next revision of the design is shown in Figure 4-1(c). The axis of the translating link has been shifted to the right and the c-space recomputed. It can be seen that the design change has produced a significant change in the shape of the c-space. The initial state of the mechanism has been located and once again marked as a. In this design a much larger increase in the value of the $\mathbf{X}$ is required before the spring can push $\Theta$ to it's extreme value. This corresponds to the segment ab in Figure 4-1(c). This solves the problem of sensitivity of to vertical displacements but the segment bc marked in the figure is still caused by a point contact and will cause sparking.

For this design, when the circuit is broken $L 2$ reaches point $\mathbf{d}$ along $\mathrm{cd}$. When the device cools off $X$ decreases and the state $e$ is reached. It is from this position that the device must be reset. One possible path for this, efcba is shown in Figure 4-1(c).

To rectify the sparking problem, a new design with the chamfered ends shown in Figure 4-1(d) is proposed. The path followed during the duty cycle of the mechanism is abc. It can be seen that the state first moves along the vertical edge ab in Figure 4-1(d). This corresponds to face contact between the two links. When the vertex at the top of that edge is 
a)

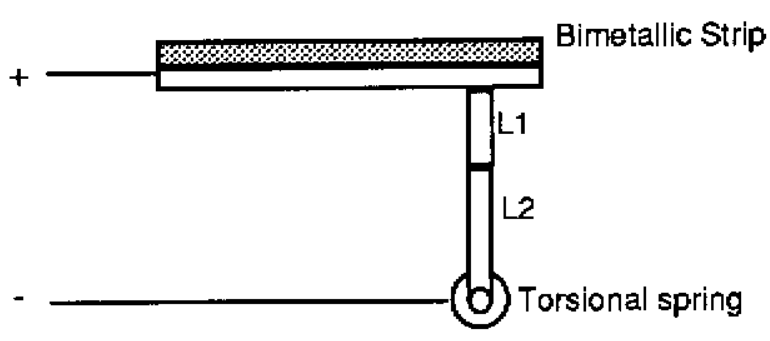

b)
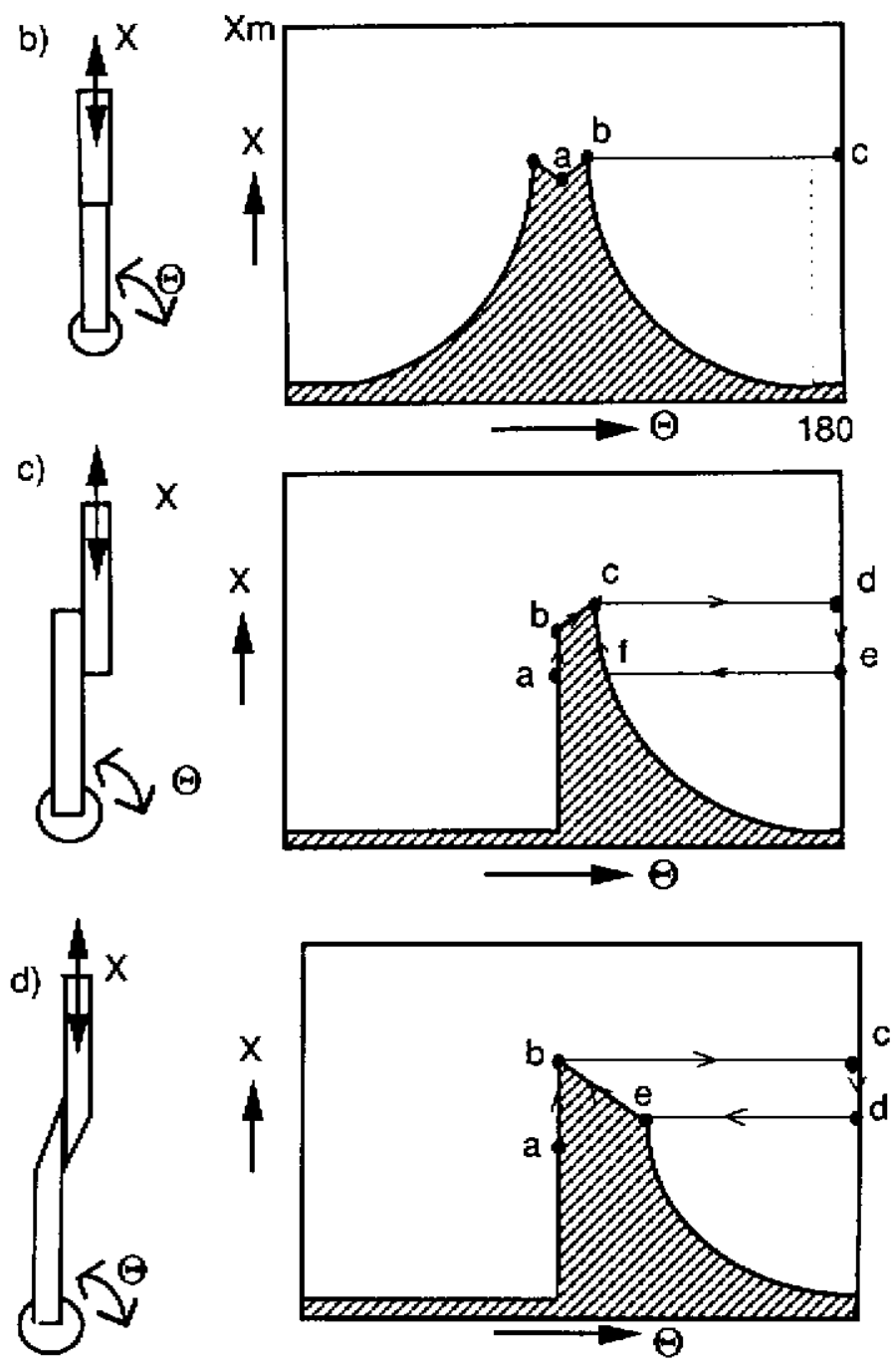

Figure 4-1: Example 1: Circuit Breaker 
reached, the contact is abruptly broken and the only point contact corresponds to vertex $b$. Hence the mechanism is seen to satisfy both of the design requirements. The path followed when the circuit is broken is abc. A possible reset path deba is also shown.

The critical tolerances on the device can now be determined. The parameters considered are the thickness of the two links and the positioning of the axis of the translating links. When these variations are made and the c-space is recalculated a drastic change in the shape of the c-space is seen. The c-space appears similar to that of one of the rejected designs. The reason for this is that the alignment of the links has a strong effect on the behavior of the device. It is therefore possible to infer that these dimensions affecting alignment are important for the functioning of the device.

To investigate the effects of wear on the device, the entire illegal region of the c-space is slightly reduced (this corresponds to a widening of the legal region of the configuration space) and all sharp comers are rounded out. One effect on the mechanism is that the increase in $X$ required before to trigger the device is reduced. The original sharp change in the contact state at b has now become a brief segment of point contact. This can lead the designer to recommend that the tip of links 1 and 2 be made harder and more wear resistant and that an adjustment device be provided to move the rest position of link 1 lower as the device wears out.

This example has shown some of the important uses of the c-space in troubleshooting a design. We were able to observe a major change in the shape of the c-space as the result of a geometric change, and minor changes as the result of variation in dimensions of parts and routine wear. A desired path was defined and the c-space constraints used to evaluate the suitability of the path.

\subsection{Example 2: Window Regulator Mechanism}

A mechanism used in automobiles to raise and lower windows is shown in Figure 4-2. This mechanism has been used as a design case study by the Engineering Design Research Center, Carnegie Mellon University. It consists of a small gear (gear 1) rotated by a handle (not shown) and meshed with a partially geared segment (gear 2) that is linked to the bottom of the window pane. When the handle is turned, gear 1 rotates and causes gear 2 to raise the window. 


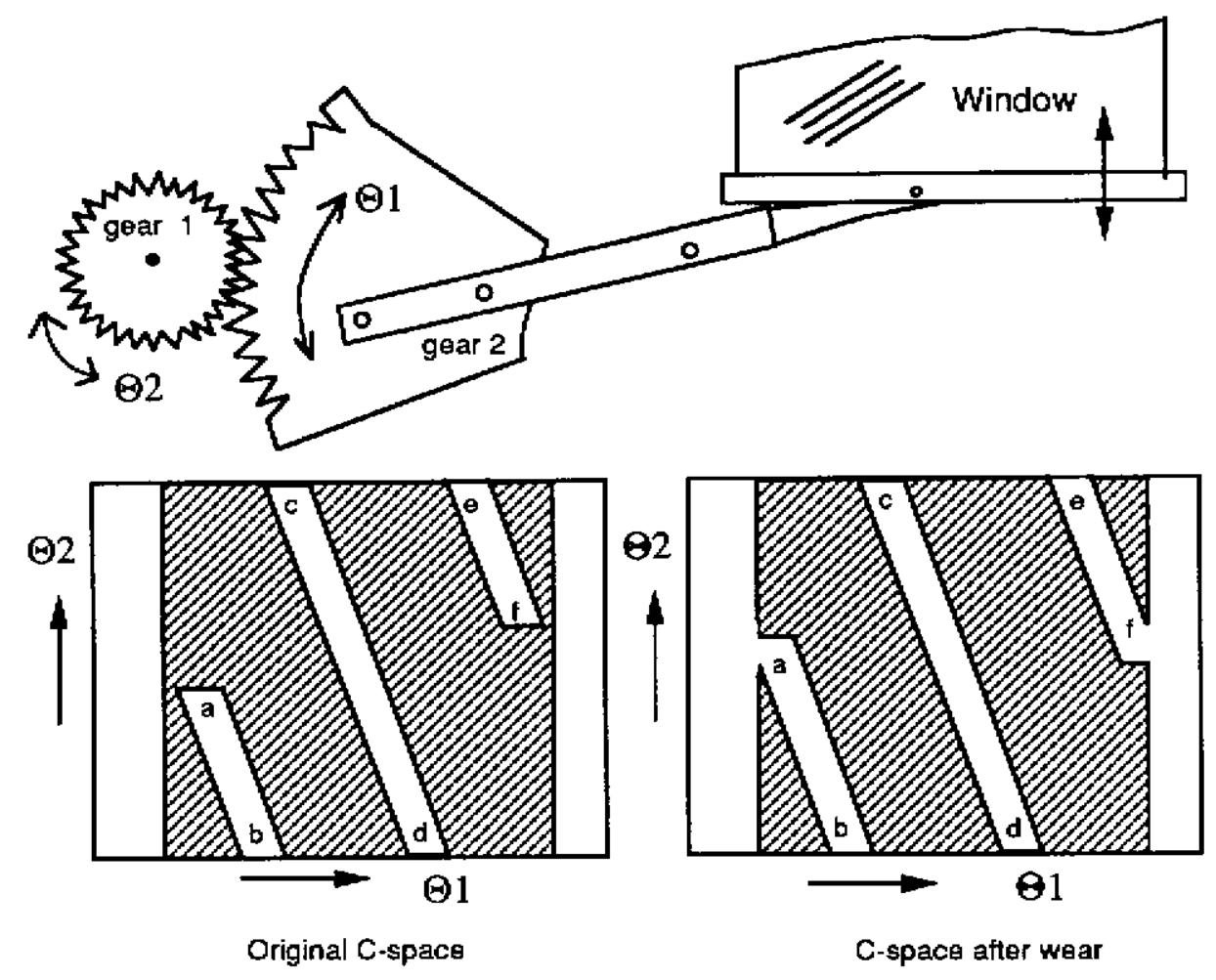

Figure 4-2: Example 2: Window Regulator Mechanism

This example examines the effects of wear on positional parameters. The operation of the mechanism involves passing sequentially through the states a,b,c,d,e and $\mathbf{f}$ and then repeating this sequence in the reverse order. Parts of the mechanism that are prone to wear are identified by two heuristics. These are follows,

1. Wear occurs along all the boundaries of the region in which the motion is occurring. This is seen as a progressive widening of the unshaded areas of the original c-space shown in Figure 4-2.

2. The wear is more severe if there are sharp changes of slope in the boundaries of the c-space. This would occur in the regions labeled $\mathbf{a}$ and $\mathbf{f}$ in the c-space Figure 4-2. 
Once these regions are identified, the corresponding features of the device are modified to account for the wear. The c-space must then be recomputed to confirm that no changes in the qualitative behavior have occurred as a result of the modification.

The effect of wear is shown by the c-space drawn after wear has occurred. The two, initially distinct, unshaded regions of the c-space have merged into a single connected region. This shows that if wear occurs, gear 2 will be able to rotate freely and the device will no longer raise the window. As the c-space boundaries responsible for this change are known, the corresponding geometric features on the device can also be identified. Steps may then be taken to make those particular areas of the part thicker or more wear resistant.

\section{Implementation Issues}

For the mode of analysis that we have used in these examples to be practical, the following capabilities are required,

1. Support for topological and geometric descriptors of the c-space.

2. Rapid generation of the c-space from the geometry and motion specification.

3. Heuristics for detection and explanation of undesirable changes in the c-space.

We now describe a computer implementation that attempts to provide the above functionality.

\subsection{Representation}

The topology and the geometry of the $c$-space are most relevant to our analysis. The topology of the c-space gives a qualitative description of the behavior of the device. For example, the number of distinct legal regions of the c-space indicates the number of distinct classes of motions that the mechanism can exhibit. Therefore, a change in the topology indicates a fundamental change in the behavior. An example of this is the window regulator mechanism that we had presented earlier (Figure 4-2).

Though topology and high level geometric parameters provide a rapid and inexpensive way of specifying a class of behaviors, they are not sufficient for a detailed analysis. The designer may want to know if a mechanism can ever attain a specific value of $x$, and this information is not available from a topological description. Hence, it is also necessary to store the exact geometry of the c-space to answer such questions. 
We are using the Noodles solid modeling system for this task (see Gursoz and Prinz [10] for details on Noodles). It provides all the functions of a standard boundary representation (brep) based modeler. It also has the additional capability of handling legal solids and other entities such as dangling edges or faces and isolated nodes in a uniform manner. This is possible because it explicitly extracts and stores detailed information about the connectivity of the different regions of the object. The topology is stored explicitly in the model and the user can query the system for this information. This is useful for detection and diagnosis of topological changes. Since the b-rep scheme is used, other information such as number of c-space boundaries etc. is also easily available. Additionally, though a minor convenience, the same representation is used for objects and c-spaces.

\subsection{C-space calculation}

Current approaches to this problem attempt to solve the problem analytically. Knowing the equations for the boundaries of the two objects and their motion paths, it is possible to formulate constraints governing possible contacts between the two bodies and to calculate them using symbolic algebra. The computation required for this problem rises with the complexity of the object boundaries and the number of degrees of freedom the problem. Hence, even for two degree of freedom cases, the process can be expedited only by restricting the scope to fixed-axis mechanisms and using simple heuristics to avoid complex calculation. This has been suggested by Joskovicz [13].

The approach that we apply for two dimensional c-spaces is to calculate the configuration space by simulation. Each object is represented as a bitmap, and motion is simulated by changing the location of these objects in a bit plane. A simple bisection algorithm is used to detect the precise point of contact. This approach has certain advantages including,

1. It is possible to obtain rapid results using special purpose hardware e.g. boards developed for computer vision work. For two dimensional cases, the computation required to calculate the configuration space at a given resolution is independent of the complexity of the object.

2. The granularity of the simulation can be controlled so that results of the desired precision are obtained. The simulation may further be limited to specific orientations of the components which are of interest to the designer.

3. Symmetry of the objects can be exploited to further reduce the amount of computation. For example, analysis of a gear pair is reduced to simulation of contact between a single pair of teeth. 
4. Intelligent strategies can be used to update previously calculated spaces rather than recomputing them entirely. This is possible because we can keep track of all the c-space boundaries affected by a particular geometric feature. If that feature is modified we only need to re-simulate all the contact points involving that feature. All others remain unaffected.

It is not possible to work with bitmaps when the c-space has a dimension exceeding two. Intersection of full three dimensional parts must be performed using the solid modeler. This extension of the simulation approach to the domain of three dimensional objects seems reasonable. This is because translation and rotation are relatively inexpensive operations in solid modeling when compared to computation of intersections. This approach only requires that interference between objects be detected and hence can be performed rapidly. However strategies to identify the most interesting degrees of freedom of a part are required.

The major problem encountered in applying this approach has been choosing the correct resolution to capture all the interesting features of a c-space. When a low resolution is being used, small changes in the geometry of the object will not be reflected by changes in the c-space. It is therefore necessary to develop strategies to estimate the proper resolution to be used. This involves deciding whether a geometric change is likely to be significant and then choosing a proper resolution to reveal the change.

The analytical approach for calculating c-spaces provides detailed equations of the c-space boundaries in terms of the mechanism dimensions. Hence, it is possible to evaluate the relative effect of different dimensions on a particular boundary of the c-space. Joskovicz [13] creates a c-space boundary map to relate geometry and c-space constraints. This records, for each boundary segment, information about the geometric features that produced it and c-space changes that will occur if these features are removed. Availability of these equations also facilitates simple analyses such as using linearized forms of constraints for approximate work. Using simulation, it is possible to record high level information about the dependencies of c-space boundaries on features. However, the exact algebraic relations are not retained. As a result, any perturbation analysis required must also be done by simulation. To overcome this handicap, we may consider calculating exact boundary equations in special situations.

The speed with which c-spaces can be updated and recomputed is a critical factor in making this a useful system. This suggests that some user-interaction may be necessary for construction of configuration spaces in complex cases. This could yield substantial 
improvements in speed since even inferences that seem trivial to the user often require considerable computation. However as it may limit the use of the analysis tool, this trade-off must be carefully evaluated.

\subsection{Reasoning}

At the most abstract level, the reasoning module should be able to detect significant changes in the c-space. Some of these queries are supported by the solid modeler, and can be easily answered. Once the information has been obtained, it must then be analyzed. The reasoning module must connect these changes to geometric features of the device. Perturbation of particular dimensions may be explicitly requested by the designer or, based on a c-space, the module could suggest a set of candidate dimensions.

The heuristics used to detect problem areas in the c-space are currently quite limited but still can handle a variety of cases. Some sample heuristics are,

- Look for regions of the c-space that are almost co-incident.

- Look for short boundary segments.

- Look for intersecting boundary segments that are almost parallel.

These regions may be further investigated by relaxation of the concerned c-space boundaries or perturbation of the concerned feature parameters followed by re-examination of the $c$ space. As more experience is gained with the system, the current set of heuristics will be further expanded. It may be possible to arrange the heuristics into a decision tree structure that systematically isolates and further investigates regions of the c-space based on their characteristics. This hierarchy is being investigated.

\subsection{Current Status}

A basic implementation has been completed. The program supports definition of objects in a $b$-rep format that is then converted into a format suitable for the solid modeler. The simulation is performed at a resolution selected by the user and the c-space is displayed on the screen. We are currently developing algorithms for dynamically varying the resolution based on the intermediate simulation results. Selection of test mechanisms to be used for collecting more heuristics for analyzing $c$-space changes is in progress. 


\section{Research Issues}

The idea of applying c-spaces to reason about tolerances, though quite promising, requires an infrastructure before it can become a usable tool. Some of the important issues include,

1. Analysis of more complicated cases

The complexity of the c-space requires that the analysis consider only two degrees of freedom at a time. A graphical interpretation is possible only in cases with two degrees of freedom or less. However we can analyse mechanisms with many degrees of freedom by considering all the kinematic pairs individually and then using the composition operator.

An important issue is whether this is a usable technique for analysis and design. Currently analysis of mechanisms is performed by analyzing kinematic pairs and propagating the constraints until the entire mechanism has been analyzed. Hence, a computer implementation providing a similar framework with additional decision support facilities can gain acceptance.

When provided with a mechanism, it is necessary to decide on the degrees of freedom that are most pertinent to the task at hand. By considering only this relevant subset of the c-space, it may be possible to analyze even complicated mechanisms efficiently.

2. Generalization of $\mathrm{c}$-space to include non-kinematic information

As currently used, the c-space axes correspond only to translation and rotational parameters. In practice, this cannot serve as a complete description of design behavior. The representation, as such, does not place any restrictions on what can be considered a valid degree of freedom. So, in the general case, variables like load and input force ranges or even temperature can be included. This generalized space could, in theory, be used as an accurate descriptor of designer intent.

If such a multi-dimensional space was maintained, it would be possible to check the relation between any two variables in the design by taking an appropriate two dimensional slice of the space. However, considerable research effort is necessary before we can even aspire to such feats.

\section{Summary}

The problem of allocating tolerances is important and CAD systems that provide the designer with tools to investigate the effect of tolerances on behavior are desirable. This requires a suitable representation of behavior that can support reasoning about tolerances and other manufacturing issues.

We have introduced in this paper, a technique for relating manufacturing tolerance to kinematic behavior using c-spaces. The basic theory behind the approach has been reviewed 
and several examples of its use in isolating potential tolerance problems demonstrated. These include detecting the sensitivity of a design to tolerances, comparing two similar designs based on this criterion and checking whether the tolerances used have led to acceptable variations in behavior. A basic implementation that provides a subset of these facilities has also been described. 


\section{References}

1. American Society of Mechanical Engineers. American Standard Limits for Cylindrical Parts ANSI B4.1, 1978.

2. American Society of Mechanical Engineers. Dimensioning and Tolerancing Standard Y14.5M. 1982.

3. Chase, K.W. and Greenwood, W.H. "Design Issues in Mechanical Tolerance Analysis". Manufacturing Review (1988).

4. Collins, J.A., Hagan, B.T. and Bratt, H.M. "The Failure Experience Matrix - A Useful Design Tool". Transactions of the ASME (Aug 1976).

5. Faltings, B. A Theory of Qualitative Kinematics in Mechanisms. Tech. Rept. UIUCDCSR86-1274, Department of Computer Science, University of Illinois at Urbana-Champaign, 1986.

6. Faltings, B. Qualitative Place Vocabularies for Mechanisms in Configuration Space. Tech. Rept. UIUCDCS-R-1360, Department of Computer Science, University of Illinois at Urbana-Champaign, 1987.

7. Faltings, B. Qualitative Kinematics of Mechanisms. Tech. Rept. No.88-01, AI laboratory, Department of Information,EPF Lausanne, 1988.

8. Forbus, K. Spatial and Qualitative Aspects of Reasoning abut Motion. Proceedings of the National Conference on AI, 1980.

9. Forbus, K. A Study of Qualitative and Geometric Knowledge in Reasoning about Motion. Tech. Rept. AI TR 615, MIT, 1981.

10. Gursoz, E.L. and Prinz, F.B. Node-based Representation of Non-manifold Surface Boundaries in Geometric Modeling. Tech. Rept. , Design for Manufacturability Laboratory, Engineering Design Research Center, Carnegie Mellon University, 1988.

11. Hillyard, R.C. and Braid, I.C. "Analysis of Dimensions and Tolerances in ComputerAided Mechanical Design". Computer-Aided Design 10, 3 (May 1978).

12. Hoffmann, P. "Analysis of Tolerances and Process Inaccuracies in Discrete Part Manufacturing". Computer Aided Design 14, 2 (March 1982).

13. Joskovicz, L. Reasoning About Shape and Kinematic Function in Mechanical Devices. Tech. Rept. TR No. 402, Courant Institute of Mathematical Sciences, New York University, 1986.

14. Nielsen, P. The Qualititive Statics of Rigid Bodies. Tech. Rept. No.1354, University of Illinois at Urbana-Champaign,Dept. of Computer Science, 1987.

15. Peck, H.. Allocating Tolerances and Limits. Longmans Green and Co., 1968. 
16. Lozano-Perez, T. and Wesley, M. "An Algorithm for Planning Collision-free Paths Among Polyhedral Obstacles". Communications of the ACM 22 (1979).

17. Lozano-Perez, T. "Spatial Planning: A Configuration Space Approach". IEEE Transactions on Computers C-32, 2 (1983).

18. Requicha, A.A.G. "Toward a Theory of Geometric Tolerancing". International Journal of Robotics Research 2, 4 (1983).

19. Reuleaux, Franz. The Kinematics of Machinery: Outline of a Theory of Machines. Dover Publications, 1963.

20. Shigley, J.E. and Mitchell, L.D.. Mechanical Engineering Design. McGraw Hill book Company, 1983.

21. Taguchi, G. Off-line and On-line Quality Control Systems. International Conference on Quality Control, Tokyo, 1978.

22. Voelcker, H.B. Modeling in the Design Process. Design and Analysis of Integrated Manufacturing Systems, National Academy of Science, 1988. 